Meta

Journal des tradlucteurs

Translators' Journal

\title{
Regard critique sur la traduction au Canada
}

\section{Pierre Cardinal}

Volume 23, numéro 2, juin 1978

URI : https://id.erudit.org/iderudit/002204ar

DOI : https://doi.org/10.7202/002204ar

Aller au sommaire du numéro

Éditeur(s)

Les Presses de l'Université de Montréal

\section{ISSN}

0026-0452 (imprimé)

1492-1421 (numérique)

Découvrir la revue

Citer cet article

Cardinal, P. (1978). Regard critique sur la traduction au Canada. Meta, 23(2),

141-147. https://doi.org/10.7202/002204ar d'utilisation que vous pouvez consulter en ligne.

https://apropos.erudit.org/fr/usagers/politique-dutilisation/ 


\section{Regard critique sur la traduction au Canada*}

Dans l'expression de l'identité nationale, la langue a toujours joué un rôle capital. Aussi, par volonté d'affirmation politique de leur identité culturelle, certains pays sont-ils allés jusqu'à accorder un statut officiel à un parler vernaculaire ou à un dialecte populaire ou rural et à le normaliser pour en faire leur langue nationale. C'est le cas, pour ne citer qu'un ou deux exemples encore assez récents, de la République sud-africaine où les Boers et leurs descendants ont fait de l'afrikaans, variété régionale de néerlandais, une des deux langues nationales (avec l'anglais) des Blancs du pays. On pense encore aux Norvégiens qui ont décidé, leur indépendance acquise, de se donner une langue nationale unifiée à partir des deux principales variétés linguistiques alors employées dans le pays. Partout la langue est une institution sociale nettement caractérisée par sa fonction première : la communication entre les hommes. Il est donc normal que, dans des pays à population hétérogène comme le Canada, le moyen essentiel de communication interlinguistique qu'est la traduction se voie, lui aussi, promu au rang d'institution.

Dans tous les États multiethniques, les rapports linguistiques entre les groupes en présence passent en effet, presque inévitablement, en bonne partie par la traduction. Tout d'abord de façon spontanée : on a alors affaire à la traduction dite naturelle, celle qui se pratique en général spontanément entre bilingues. Les rapports et échanges entre les ethnies en présence augmentant souvent rapidement, la traduction s'institutionnalise très vite. Il semble bien que le Canada, composé dès ses origines de deux groupes majoritairement unilingues en contact l'un avec l'autre, se soit trouvé très tôt dans cette situation. Les historiens de la traduction nous apprennent en effet que, dès ses débuts, la Nouvelle-France connut des traducteurs ${ }^{1}$ : il s'agissait simplement, bien sûr, de colons qui servaient de truchements entre Français et Amérindiens dans des buts essentiellement commerciaux. I1 est donc intéressant de noter en passant que, déjà à cette époque, ce sont les Canadiens, les « Français », minoritaires,

\footnotetext{
* Une première version de cet article a été communiquée oralement au troisième colloque international "Identité culturelle et francophonie dans les Amériques 》, tenu à Toronto du 2 au 5 juin 1976. La présente version date d'août 77. Nous ne croyons pas que la situation fondamentale qui y est décrite se soit modifiée depuis.

1. On trouvera un précieux historique partiel de la traduction au Canada in Meta 22, 1, mars 1977, (numéro spécial : Histoire de la traduction au Canada). Pour la genèse de l'institution sous le régime français, voir l'article de Jean Delisle, p. 5-14; celle de la «politisation» de l'institution dès après la conquête anglaise se dégage des articles de Paul Horguelin, p. 15-25, Jacques Gouin, p. 26-32 et Fikri Méléka, p. 57-65.
} 
qui étaient forcés de pratiquer un certain bilinguisme, les Indiens longtemps majoritaires dans les territoires partagés pouvant se permettre de rester unilingues. Sous le régime français, bien qu'institutionnalisée, la traduction resta cependant une activité à but essentiellement économique ; d'indispensables bilingues faisant le pont entre les deux peuples en présence l'exerçaient.

Ce n'est qu'après la conquête anglaise que la traduction deviendra un des rouages, bien modeste d'abord, de l'administration publique. Les contacts entre les nouveaux administrateurs anglais militaires, puis civils, et les «Canadiens 》 moyens furent d'abord assez peu nombreux. Rapidement, cependant, le nombre de ces administrateurs, bientôt suivis de commerçants, augmenta ; et grâce à la présence prépondérante de ceux-ci dans la vie politique et commerciale de la colonie, l'anglais pris vite la place qui revenait à la langue des nouveaux maîtres : la première. À partir de ce moment les Canadiens - qui deviendront bientôt les Canadiens français - feront de plus en plus les frais d'un nouveau bilinguisme, la plupart du temps à sens unique, situation qui paraît tout à fait normale chez un peuple conquis par les armes et au territoire occupé. Peu après le début du régime anglais donc, la traduction revêtait déjà la forme d'une institution à caractère politique.

Effacée au début et sans structure précise, la traduction imposera sa présence partout où des contacts se produiront entre les membres de ce qui deviendra vite deux communautés ethniques nettement identifiées. Cette traduction sera longtemps faite par des gens pleins de bonne volonté - et souvent d'ailleurs non dépourvus d'honnêtes connaissances générales et linguistiques - mais sans compétence particulière : journalistes, greffiers, commis aux écritures, hommes de loi, etc., sans oublier les inévitables secrétaires «bilingues». Dans les premières décennies de la Confédération canadienne, on ne compte cependant à la fonction publique fédérale qu'une poignée de traducteurs, qui ne recevront de reconnaissance officielle qu'au début du $20^{\mathrm{e}}$ siècle. Mais, en 1934, lors de la création du Bureau (fédéral) des traductions et la nomination d'un «Surintendant $»$, on y dénombre déjà une équipe de 91 traducteurs ${ }^{2}$. Avec le réveil du nationalisme canadien-français dans les années 40 et 50 et les soubresauts de la «Révolution tranquille » québécoise des années 60 , la «bilinguisation 》, à défaut de la «biculturalisation », officielle du pays devient inévitable. C'est à partir de ce moment que, de rouage administratif qu'elle était - rouage utile mais obscur, négligé et, disons-le, pas toujours apprécié - la traduction devient une profession socialement visible et politiquement indispensable : en 1965, en l'An V de la «Renaissance » canadienne-française, le nombre de traducteurs fédéraux en titre s'élève déjà à environ trois cent cinquante. Le besoin de traducteurs se fait si impératif qu'en 1968 le Bureau fédéral des traductions parrainera un programme de formation universitaire à l'intention d'un certain nombre de boursiers choisis par lui. Fait important à remarquer, cependant : toutes ces bourses sont exclusivement réservées à des candidats qui ont le français comme langue de travail. Les résultats de ces efforts de recrutement ne se font pas longtemps attendre et, en 1977, le Bureau peut compter sur un

2. Voir F. Méléka, op. cit., p. 59. 
contingent de près de treize cents traducteurs. Ces données, purement quantitatives, demandent à être interprétées.

Ce qui nous semble révélateur ici, c'est la répartition du travail entre les traducteurs des deux langues. En 1977, 90\% des traducteurs fédéraux ont le français comme langue de travail, et $10 \%$ l'anglais. Ces proportions, une fois inversées, correspondent à peu près parfaitement à la langue dans laquelle l'ensemble des fonctionnaires fédéraux travaillent et rédigent leurs textes : $90 \%$ en anglais, $10 \%$ en français. Or, on sait qu'actuellement, à la fonction publique fédérale, presque les trois quarts $(72,4 \%)$ des fonctionnaires ont l'anglais comme langue première ou d'usage, et à peu près le quart $(27,6 \%)$ le français, ces pourcentages reflétant assez fidèlement la répartition \& bi-linguistique $\gg$ effective des citoyens du pays ${ }^{3}$. Ces chiffres montrent donc en clair, après quelques calculs élémentaires, que moins de la moitié (environ $40 \%$ ) des fonctionnaires fédéraux francophones rédigent habituellement leurs textes dans leur langue et que plus de la moitié d'entre eux (environ 60\%) doivent travailler - par écrit au moins - dans une langue, donc selon une mentalité, des habitudes culturelles qui ne sont pas les leurs, situation profondément inéquitable camouflée par les statistiques officielles de l'État sur la répartition des fonctionnaires selon leur langue maternelle. Malgré la volonté politique officielle de changer cet état de choses, les réformes opérées depuis une dizaine d'années dans la répartition des postes de la fonction publique fédérale entre francophones et anglophones restent surtout d'ordre quantitatif et sont encore éloignées de l'idéal à atteindre ${ }^{4}$. La fonction publique a tout simplement été «bilinguisée »: son caractère fondamentalement anglophone n'a pas subi de véritable modification. Afin qu'elle soit à l'image du pays, afin de devenir un millieu accueillant pour les membres de la minorité officielle, il faudrait qu'elle se transforme en une institution authentiquement biculturelle ${ }^{5}$. On voit mal comment les timides réformes mises en œuvre à l'heure actuelle pourraient y parvenir.

Le secteur privé de l'économie québécoise n'a pris au sérieux les besoins en traduction que depuis une date relativement récente et $n^{\prime} y$ a toujours accordé que le minimum exigé par la concurrence commerciale, quand il ne cédait pas aux diverses pressions ouvertes exercées par le «marché québécois ou par: le gouvernement provincial ${ }^{6}$.

3. Toutes ces statistiques nous ont été fournies par la direction du Bureau fédéral des traductions et par la Commission de la fonction publique du Canada et portent sur l'exercice qui s'est terminé en avril 77 . Nous exprimons notre reconnaissance aux fonctionnaires de ces deux organismes et du Commissariat aux langues officielles pour l'aide précieuse qu'ils nous ont apportée dans l'interprétation de ces données quantitatives.

4. Les chiffres suivants nous semblent éloquents à cet égard : selon le Conseil du Trésor lui-même, seulement $33,3 \%$ des fonctionnaires fédéraux francophones occupent des postes scientifiques et professionnels et $19,9 \%$ des postes de cadres. Voir le document "confidentiel » (inédit à cette date !) du Conseil du Trésor que le journal le Devoir a résumé et présenté à ses lecteurs le 11.8 .77$, p. 1 et 6 .

5. Voir à ce propos l'opinion des auteurs du rapport de la «Commission royale d'enquête sur le bilinguisme et le biculturalisme », en particulier leurs célèbres \& pages bleues ».

6. On sait qu'au Canada, le Québec est l'une des dix « provinces » fédérées et dirigées chacune d'elles, par un gouvernement possédant juridiction entière ou partielle dans des sphères d'activité délimitées par la constitution fédérale de 1867. * Province \$ doit donc y être pris au sens américain de State ou allemand de Land. 
Dans son ensemble d'ailleurs, le secteur privé québécois reproduit assez fidèlement le secteur public fédéral. Les grandes et moyennes entreprises, tributaires dans une très forte proportion d'intérêts anglo-canadiens ou américains, emploient essentiellement l'anglais comme langue de travail ?. Encore aujourd'hui, cadres, employés, contremaîtres et parfois même simples ouvriers, francophones ou allophones, doivent y connaître l'anglais au moins assez pour le comprendre. Se comportant au Québec à peu près comme partout ailleurs au Canada, ces entreprises anglophones s'y contentaient récemment encore de quelques intermédiaires bilingues - la plupart francophones ${ }^{8}$ - pour assurer leurs contacts directs avec le public francophone. La traduction devint vite indispensable à partir du moment où ces entreprises se soucièrent de protéger leur «image de marque ». Et une fois de plus la traduction - véritable baromètre de la nature des rapports qui existent entre nos deux communautés linguistiques - se fit surtout de l'anglais au français! Même la publicité destinée aux francophones du Québec est encore aujourd'hui en grande partie conçue et rédigée en anglais ${ }^{9}$, c'est-à-dire selon des habitudes culturelles et des schèmes de pensée aliénants parce qu'inconnus de la très vaste majorité d'entre eux.

Aux Québécois - relégués au rang de véritables étrangers dans leur propre pays - cette situation d'aliénation culturelle a fini par devenir inacceptable. Depuis deux ou trois ans, ils ont entrepris ouvertement et officiellement d'effectuer des transformations radicales au moins dans le monde du travail de leur province. La francisation et la «francophonisation» des entreprises anglophones seront bientôt irréversibles. La traduction continuera probablement d'y prospérer comme dans toute situation linguistique mixte, mais elle cessera d'être presque à sens unique : on en inversera la direction, comme il se doit, et c'est surtout du français à l'anglais qu'elle devrait se faire dans un avenir vraisemblablement assez proche ${ }^{10}$.

Depuis une décennie, le rôle de la traduction au Canada a beaucoup évolué. Et si on examine d'un ail critique la nature des changements effectués, on peut faire les deux grandes constatations suivantes :

7. Selon une enquête récente (le Devoir, 30.6.77, p. 4) du Montreal Board of Trade, seulement $31 \%$ des cadres supérieurs et $39 \%$ des cadres moyens des sièges sociaux établis au Québec étaient des francophones en 1977, cela malgré des progrès notables depuis dix ans. On devine l'effet de cette prépondérance des anglophones sur ces milieux de travail. Le lecteur désireux de mieux comprendre la résistance au changement opposée par ces entreprises grâce à leur structuration sociale et ethnique consultera avec profit les ouvrages de Porter et Clemens ainsi que l'article de ce dernier dans Impetus (voir notre bibliographie).

8. Le dernier rapport annuel de la Régie de la langue française du Québec signale (p. 144) que $60 \%$ des non-francophones du Québec ne peuvent utiliser la langue française de façon courante. Ces derniers forment une minorité de près d'un cinquième de la population du Québec.

9. Selon le publicitaire très connu Maurice Watier, cité par J. Delisle in Meta, 22, 1, p. 70 De façon plus précise, ce dernier estime qu'« au moins $70 \%$ des annonces de presse et télévisées diffusées [en français] au Québec sont des traductions [de l'anglais]... 》 (Ibid.). On trouvera une intéressante étude sociologique de la question chez Frederick Elkin (voir la bibliographie)

10. Jusqu'à présent, cependant, peu de choses semblent avoir commencé à changer. Selon une interview qu'il accordait récemment à un journal anglophone de Montréal, l'actuel président de la Société des traducteurs du Québec estime que, dans la plupart des entreprises du Québec, seulement 10 pour cent des traductions se font du français à l'anglais. Les autres langues comptant pour très peu, on constate donc que, même au Québec, le rapport 90-10 en faveur de l'anglais ne fait que confirmer la tendance générale du pays. 
Primo : la traduction est devenue une véritable institution publique omniprésente - ou presque - dans notre pays, institution qui :

a) est articulée par trois associations ou sociétés professionnelles provinciales (au Nouveau-Brunswick, au Québec et en Ontario) chapeautées par un conseil pan-canadien ;

b) compte sur une véritable armée de quelque 2000 traducteurs ${ }^{11}$, c'est-àdire 1 traducteur pour 10000 citoyens environ, ce rapport passant à 1 traducteur pour environ 2500 citoyens si l'on tient compte du fait que neuf traducteurs sur dix sont francophones, et que neuf traductions sur dix sont faites en français pour le public francophone ( $26 \%$ de la population du pays) ;

c) est « approvisionnée » par six programmes de formation professionnelle menant à un grade universitaire - tous donnés par des établissements d'enseignement francophones ou bilingues - sans parler des quatre ou cinq autres programmes de formation à temps partiel ; cette pléthore de programmes intéressent près d'un milier d'aspirants traducteurs, le tout essentiellement pour un petit marché de sept ou huit millions de personnes $! \AA$ notre connaissance une telle situation est unique au monde.

Secundo : de par son évolution récente, la traduction est à l'heure actuelle un véritable miroir linguistique des rapports sociopolitiques institués entre les membres des deux communautés linguistiques officielles du pays :

a) l'institution a existé jusqu'à maintenant presque uniquement pour communiquer à des francophones le contenu de textes de toutes sortes conçus et rédigés en majeure partie pour et par des anglophones;

b) cette situation se répète chaque fois que les deux groupes linguistiques sont en présence et elle est particulièremnt flagrante à la fonction publique fédérale et, à quelques exceptions près, dans toutes les entreprises privées québécoises qui ne sont pas la propriété de francophones.

Il se dégage donc une impression d'ensemble assez nette du bref tableau historique et descriptif que nous venons de tracer : du point de vue auquel nous nous sommes placé, il existe au Canada deux sociétés, deux communautés linguistiques officielles, dont l'une, l'anglophone, est essentiellement la société traduite, et l'autre, la francophone, la société traduisante. La société traduite est la société politiquement dominante, celle qui a pu, par conséquent, rester effectivement unilingue; la société traduisante est celle qui est dominée et qui a dû, à ce titre, devenir bilingue. Même si on nous objecte que cette situation est le produit de nombreux facteurs historiques (comment pourrait-on ne pas l'être!) et qu'elle semble en voie de transformation «officielle» sinon universellement effective ${ }^{12}$, cela ne change rien à la nature des rapports sociopolitiques qui ont

11. Sans parler des innombrables secrétaires, imposteurs, charlatans et autres amateurs à qui, « puisqu'ils sont bilingues », on confie des textes à traduire! Pour donner au lecteur un ordre de grandeur, au seul Bureau fédéral, plus de 232 millions de mots ont été traduits dans la seule année 76-77.

12. Sur la dose certaine de scepticisme et de patience avec laquelle ces réformes doivent, jusqu'à nouvel ordre, être accuillies, voir les remarquables rapports annuels de M. Keith 
existé jusqu'à aujourd'hui entre ces deux communautés nationales et qui sont essentiellement des rapports de force.

La traduction est devenue une institution-tampon entre nos deux communautés nationales. Elle vise à donner à la société traduisante, la francophone, l'illusion d'une participation officielle à la vie du pays tout entier alors que ce sont les membres de la société traduite, l'anglophone, qui y occupent effectivement une place disproportionnée. "L'institution traduction» joue donc un rôle éminemment politique - bien qu'en apparence modeste - dans la vie du Canada. C'est vraisemblablement à son corps défendant que l'institution assume ce rôle politique, pour ne pas dire idéologique ${ }^{13}$, sa fonction première, sa vraie nature pourrait-on dire, étant sociale mais apolitique : permettre à des gens d'une langue de comprendre des gens d'une autre langue avec qui ils entretiennent, idéalement, des rapports d'égalité. Malgré ces circonstances atténuantes pour ceux qui la pratiquent, il n'en reste pas moins que la traduction a vu sa fonction sociale propre se transformer au Canada, particulièrement dans les services de l'État fédéral dont on serait en droit d'attendre qu'il y régnât la plus parfaite égalité possible. On sait malheureusement qu'il n'en est encore rien, en dépit de la bonne volonté officielle. C'est en cela que la traduction est une véritable institution-miroir de la situation collective du Canada français. Dans de telles circonstances, il est permis de mettre en doute, pour le moment du moins, une grande partie de la supposée contribution de la traduction au maintien de notre identité culturelle et de nos droits à l'égalité des chances. Seules des réformes en profondeur pourraient renverser le courant actuel. C'est à cette condition seulement que la traduction assumera chez nous le noble rôle qui lui revient dans toute société normale : celui d'institution privilégiée de communication bilatérale entre hommes de langue et de culture différentes mais de droits égaux.

Pierre Cardinal

\section{ÉLÉMENTS BIBLIOGRAPHIQUES}

CALVET, L.-J., Linguistique et colonialisme, Paris, Payot, 1974.

CLEMENS, W., The Canadian Corporate Elite, Toronto, McClelland and Stewart, 1975.

CLEMENS, W., \& The Money and the Power » in Impetus (supplément mensuel du Financial Post), mai 1975, p. 19-22 et 36.

COMMISSION DE LA FONCTION PUBLIQUE DU CANADA, Rapport annuel 1976, Ottawa, Ministère des Approvisionnements et Services, 1977.

ELKIN, F., Rebels and Colleagues. Advertizing and Social Change in French Canada, Montréal, McGill University Press, 1973.

GOUVERNEMENT DU QUÉBEC, Régie de la langue française, Rapport annuel, 1976-77, Québec, Éditeur officiel du Québec, 1977.

Spicer, ex-commissaire fédéral aux langues officielles, plus précisément le ou les chapitres $V$ du rapport de l'année 70-71; I de 71-72; I et II de 72-73; et le chapitre I de 73-74, 75 et 76. A notre avis, ces rapports constituent la meilleure initiation qui soit aux réalités concrètes du biculturalisme canadien.

13. Au lecteur qui douterait de la possibilité d'utiliser l'institution sociale qu'est la langue à des fins idéologiques (même quand elles ne sont pas explicites), nous conseillons vivement la lecture de la sérieuse étude de L.-J. Calvet donnée en bibliographie. 
HAUUGEN, E., «Language Planning in Modern Norway in Readings in the Sociology of Language, Paris, Mouton, 1970, p. 673-687.

LAURENDEAU, A. et DUNTON, A.D., Rapport préliminaire de la Commission royale d'enquête sur le bilinguisme et le biculturalisme, Ottawa, La Commission, 1965.

LAURENDEAU, A., DUNTON, A.D. et GAGNON, J.-L., Rapports de la Commission royale d'enquête sur le bilinguisme et le biculturalisme, Ottawa, Imprimeur de la Reine, 19671971 .

LEPAGE, Robert Brock, The National Language Question, Londres, Oxford University Press, 1964.

META, 22, 1, mars 1977 (Histoire de la traduction au Canada).

POFTER, John, The Vertical Mosaic: An Analysis of Social Class and Power in Canada, Toronto, University of Toronto Press, 1965.

SECRÉTARIAT D'ÉTAT, Rapport annuel (1976-77), Ottawa, Ministère des Approvisionnements et Services, 1977.

SPICER, Keith, Commissaire aux langues officielles, Rapports annuels (de 1970-71 à 1976), Ottawa, Ministère des Approvisionnements et Services, 1971-76. 